\title{
The worldwide assessment of separation of patients from ventilatory assistance (WEAN SAFE) ERS Clinical Research Collaboration
}

\author{
Leo Heunks ${ }^{1}$, Giacomo Bellani ${ }^{2,3}$, Tài Pham ${ }^{4,5,6}$, Laurent Brochard ${ }^{4,5,6}$ and \\ John G. Laffey $\mathbb{1}^{4,7,8}$
}

Affiliations: ${ }^{1}$ Dept of Intensive Care Medicine, Amsterdam UMC, location VUmc, Amsterdam, The Netherlands. ${ }^{2}$ School of Medicine and Surgery, University of Milan-Bicocca, Monza, Italy. ${ }^{3}$ Dept of Emergency and Intensive Care, San Gerardo Hospital, Monza, Italy. ${ }^{4}$ Keenan Research Centre for Biomedical Science, St Michael's Hospital, Toronto, ON, Canada. ${ }^{5}$ Dept of Critical Care Medicine, St Michael's Hospital, Toronto, ON, Canada. 'Interdepartmental Division of Critical Care Medicine, University of Toronto, Toronto, ON, Canada. ${ }^{7}$ Anaesthesia, School of Medicine, National University of Ireland Galway, Galway, Ireland. ${ }^{8}$ Regenerative Medicine Institute (REMEDI) at CÚRAM Centre for Research in Medical Devices, Biomedical Sciences Building, National University of Ireland Galway, Galway, Ireland.

Correspondence: John G. Laffey, Anaesthesia and Intensive Care Medicine, School of Medicine, National University of Ireland Galway, Costello Road, Newcastle, H91 TK33, Galway, Ireland.

E-mail: john.laffeyanuigalway.ie

\section{@ERSpublications}

The worldwide assessment of separation of patients from ventilatory assistance (WEAN SAFE) Clinical Research Collaboration is addressing key issues related to weaning from mechanical ventilation http://ow.ly/lggq30nRSWG

Cite this article as: Heunks L, Bellani G, Pham T, et al. The worldwide assessment of separation of patients from ventilatory assistance (WEAN SAFE) ERS Clinical Research Collaboration. Eur Respir J 2019; 53: 1802228 [https://doi.org/10.1183/13993003.02228-2018].

\section{Rationale}

Successful weaning of patients from invasive mechanical ventilation (IMV) represents a critical hurdle in the recovery process following severe respiratory failure [1-3] and is a key clinical challenge for intensive care unit (ICU) clinicians. Many of the serious complications of IMV are directly related to the duration of ventilation $[4,5]$. Failure to successfully separate patients from IMV contributes directly or indirectly to poorer patient outcomes including, of course, longer duration of ventilation, longer length of ICU and hospital stay, and higher patient morbidity (dyspnoea, infection, muscle weakness) and mortality $[6,7]$. Some patients spend a considerable amount of time in the process of being liberated from IMV. The identification and utilisation of approaches that improve the success of ventilator weaning are therefore of fundamental importance [8-10].

Despite the importance of the weaning period, this process is not rigorously defined, with wide variations in definitions and practices. In addition, the specific impact of ventilator weaning difficulties on patient outcomes is still poorly understood and little is known regarding the impact of physical and mental conditions, including frailty, before ICU admission and comorbidities (e.g. chronic obstructive pulmonary disease or heart failure) on weaning outcome. While guidelines do exist on the classification of weaning [1], a key recent study has shown that these are not applicable to all patients [11]. Moreover, different 
practices exist in regard to weaning procedures and some confusion exists even in what should be considered the beginning of the weaning process. This is an important problem, because general recommendations regarding the entire weaning process may encompass completely different causes and consequences of its prolongation, and therefore may be totally inappropriate for individual patients. The diversity of factors contributing to difficult/prolonged patient weaning from ventilation mean that management must be individualised based on the specific underlying contributing factors.

The WorldwidE AssessmeNt of Separation of pAtients From ventilatory assistancE (WEAN SAFE) study is an initiative of the acute respiratory failure section of the European Society of Intensive Care Medicine (ESICM), which endorsed the study, and has been supported by Assembly 2 (respiratory intensive care) of the European Respiratory Society (ERS). The ERS endorsed it as a Clinical Research Collaboration (CRC), expanding the scope of the project. WEAN SAFE will aim to address key issues relating to weaning from IMV. The ERS CRC programme provides support to projects in different areas of respiratory medicine, to build and maintain pan-European and/or global collaborations [12]. The rationale for forming a WEAN SAFE CRC as a component of this large-scale project was to harness the resources of a CRC for a project that has a broad reach across respiratory intensive care and requires the collaboration of ICUs across the globe. This will enable WEAN SAFE to generate and analyse a large geographically diverse cohort of patients receiving IMV, using an unbiased approach that does not attempt to pre-define "weaning" but instead simply enrols all patients who have required at least 2 days of IMV.

\section{Aims}

The over-arching aims of WEAN SAFE are to describe, in a "real world" global cohort of ICU patients, the current procedures for weaning and the applicability of existing weaning classification systems, and to identify factors relating to patients and their healthcare environment that are associated with delays in weaning from IMV. Table 1 lists key pre-defined "unanswered questions" that the WEAN SAFE project will address.

In order to generate a database of sufficient size and scale, a "convenience sample", of over 5000 patients was targeted. Based on the LUNG SAFE (Large Observational Study to Understand the Global Impact of Severe Acute Respiratory Failure) data [13], we estimated that a medium-sized ICU would enrol approximately 11 patients invasively ventilated on day 2 following intubation per participating ICU over a 4-week period. Given this, we therefore targeted the enrolment of 500 participating ICUs (considering a $10 \%$ dropout) to reach the patient enrolment target.

\section{Past achievements}

While this is a newly established CRC, the members of the WEAN SAFE CRC executive committee have a strong track record in establishing research collaborations and of large-scale epidemiological research in respiratory intensive care, having previously founded and led the LUNG SAFE global collaboration. The LUNG SAFE project had a similar structure to the WEAN SAFE CRC. For LUNG SAFE, we were successful in recruiting 459 ICUs across 50 countries globally. The LUNG SAFE database is the largest epidemiological study of acute respiratory distress syndrome and acute hypoxaemic respiratory failure to date, with over 29000 patients screened and data collected from over 12000 patients. The principal

\footnotetext{
TABLE 1 Unanswered questions to be addressed by the worldwide assessment of separation of patients from ventilatory assistance (WEAN SAFE) study

$1 \quad$ What is the frequency of delayed weaning from IMV?

$2 \quad$ What current approaches are taken to wean patients from IMV?

$3 \quad$ What factors are used to determine when patients are in the weaning phase?

$4 \quad$ What are the barriers to effective weaning from IMV?

$5 \quad$ What are the factors (patient, institutional, medical practice) that contribute to failed attempts to wean from IMV?

$6 \quad$ What is the impact of sedation management on weaning from IMV?

$7 \quad$ What is the impact of pre-morbid conditions and of frailty on weaning from IMV?

$8 \quad$ What is the utility of existing classifications for weaning from IMV?

$9 \quad$ What is the impact of early versus delayed and/or failed weaning from IMV?

10 What is the role of noninvasive ventilation in the weaning process?

11 What regional or geo-economic differences exist regarding weaning from IMV?

12 What is the therapeutic resource use in patients with delayed weaning from IMV?
}

IMV: invasive mechanical ventilation. 
manuscript [13] was published in JAMA, while 10 additional papers have been published and several more papers are in preparation.

\section{Current status}

WEAN SAFE successfully leveraged its existing global ICU network (formerly the "LUNG SAFE" Investigators) to develop the WEAN SAFE Investigator group. Centres were recruited through assembly meetings of the ESICM and ERS, personal communication and websites. The protocol for the WEAN SAFE prospective cohort study was developed, and an electronic case report form generated. The enrolment window for the WEAN SAFE prospective cohort study consisted of any four consecutive weeks between October 1, 2017, and May 31, 2018, and the study completed follow-up on August 31, 2018. The study population comprised patients that still required IMV $48 \mathrm{~h}$ after initiation of IMV (or $48 \mathrm{~h}$ after ICU admission for patients already receiving IMV). Exclusion criteria comprised patients in whom consent was not obtained (in centres where this was a requirement of the local ethics committee).

It has been recognised that patient and family engagement is important in clinical care and research related to the ICU and specifically in the field of weaning from IMV [14]. The European Lung Foundation, an organisation that brings patients, the general public and medical professionals together, is a partner in this CRC, and has nominated a patient representative to the CRC steering committee. This partnership will enable us to define meaningful patient-centred outcome parameters, enable dissemination of new knowledge derived from the WEAN SAFE project among patient organisations, and help prioritise future directions for this CRC.

The data for this observational study were collected as part of routine clinical care and are anonymised. For many participating sites, informed patient consent was not deemed necessary by local research ethics boards. However, there was considerable variation by country in regard to this aspect of the consent procedure, with a significant proportion of sites requiring informed consent by the patient or a substitute decision-maker. Each site investigator was ultimately responsible for obtaining approval from their relevant ethics committee, in compliance with the local legislation and rules. In most participating countries, a national coordinator liaised with the participating centres and assisted with the ethical approvals process.

In total, 456 centres from 52 countries participated in WEAN SAFE (figure 1a), with over 12000 patients screened for enrolment. The top five recruiting countries, namely Italy, Spain, the USA, France and the UK each screened over 700 patients (figure 1b). Of the 12000 patients screened, over 5000 patients met the criteria for enrolment into the WEAN SAFE database, i.e. these patients required IMV for at least two calendar days following tracheal intubation. As this is a "convenience" sample, derived from ICUs that voluntarily participated, a significant limitation is that there will be significant geographic imbalances in the dataset. While the primary analysis will focus on the entire global dataset, subsequent analyses will examine the impact of geographic and economic issues, as was done previously in the LUNG SAFE dataset [15].

\section{Future plans}

We are currently validating data entered into the WEAN SAFE database. The target date for completion and closure of the database is April 30, 2019. The target date for the primary manuscript is autumn 2019. From all centres that enrolled patients, two investigators will be listed as collaborating authors in the primary and major secondary publications. In addition, after the pre-specified analyses have been completed, and the data published, the data will be made available under certain conditions for all members of the current CRC for research purposes.

This established global CRC of centres interested in weaning from IMV will be important for future scientific studies and development of guidelines, task forces and other initiatives relevant to this topic. When initial analysis of data is completed, we will invite new centres to collaborate in this CRC. One specific population not addressed in the current study is the weaning of patients in respiratory step-down units, weaning centres or specialised respiratory units that have advanced expertise with a specific population. As the WEAN SAFE cohort study has not been specifically designed to capture this important subpopulation, this could constitute a follow-up project, and WEAN SAFE can inform the optimal design of such a project. In addition, the data from the WEAN SAFE study should constitute a useful resource to inform revised guidelines regarding weaning from IMV.

\section{Challenges}

The scale of this project, in terms of the required number and distribution of ICUs (over 500) required to generate a patient cohort of sufficient size (over 5000 patients), geographic distribution and scale, has presented considerable challenges, which have largely been successfully overcome at this point. A particular issue has been the requirement from some ethics committees for informed patient consent to be 


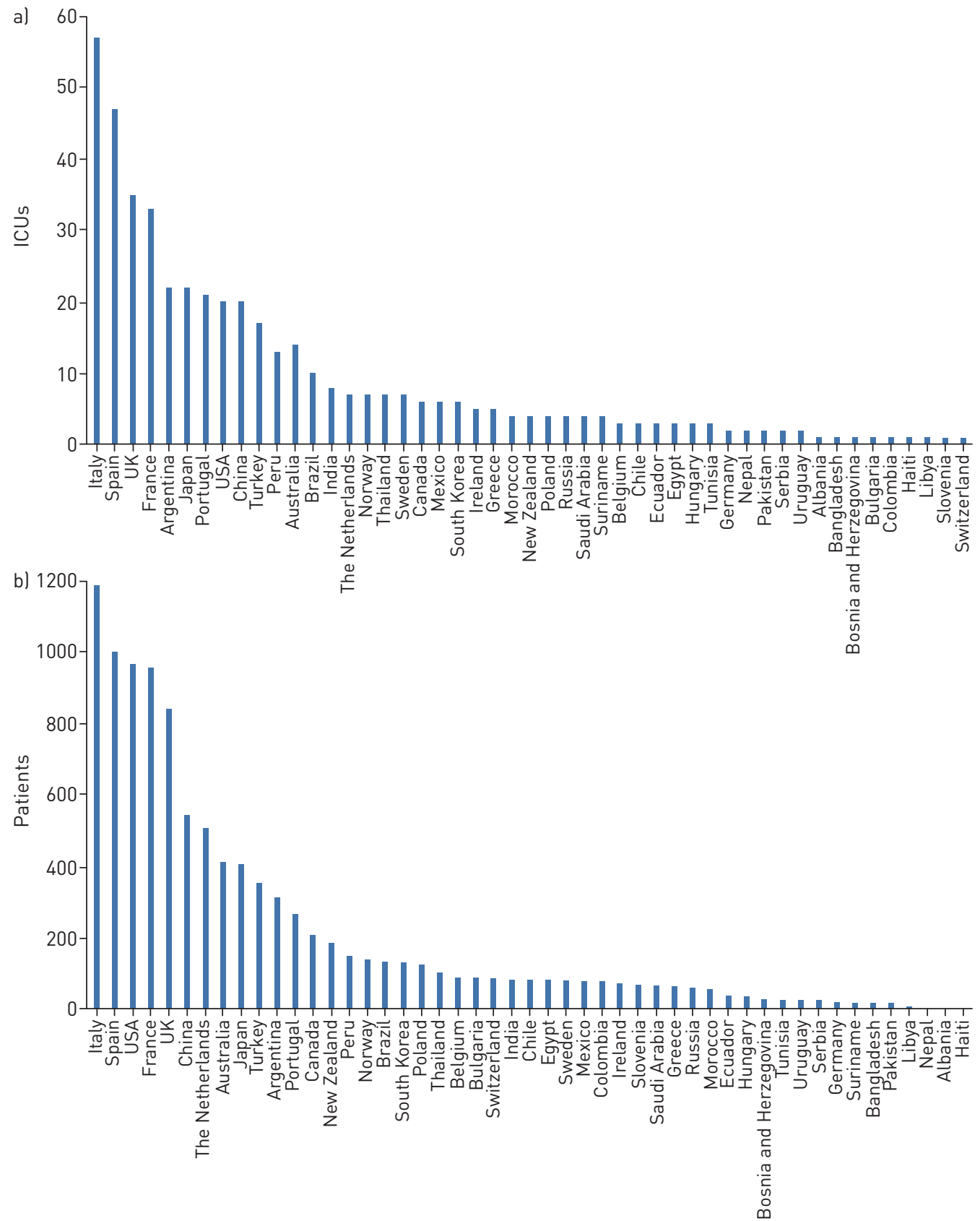

FIGURE 1 a) Participating intensive care units (ICUs) in the worldwide assessment of separation of patients from ventilatory assistance (WEAN SAFE) prospective cohort study, by country. b) Patients enrolled in the WEAN SAFE prospective cohort study, by country.

obtained for patient enrolment into our (noninterventional) study. Restrictions regarding data collection for prospective observational datasets such as WEAN SAFE are likely to increase with the recent introduction of General Data Protection Regulations across the European Union. These restrictions may skew patient enrolment into observational studies, potentially making these datasets less representative of the full population. Experiences from WEAN SAFE are an excellent opportunity to critically review the impact of these new regulations on clinical and research collaborations.

We anticipate additional challenges related to the analysis and interpretation of the WEAN SAFE database. Although there are published guidelines about when and how to start the weaning process, we do not know whether these recommendations are used or are feasible, what the barriers are for their implementation and what the real-life impact is of an early or late weaning process for the patient. There 
is also significant uncertainty about when the process of weaning from IMV is really starting, in our understanding of the impact of sedation management, and knowledge regarding current weaning practices and how this is associated with outcomes.

\section{Conclusions}

WEAN SAFE will assemble the largest database of clinically relevant data related to weaning from IMV to date, generating an unprecedented research resource. The WEAN SAFE CRC will facilitate the generation and dissemination of important insights into the diversity and impact of weaning practices and outcome in (nearly) all parts of the globe, and engage with patients and their families in this process. We look forward to the next stage of this exciting project, and to advancing the field of research into weaning from IMV to improve respiratory intensive care and patient outcomes.

Conflict of interest: L. Heunks reports grants from Orion Pharma and Vent free, and travel reimbursement for a presentation from Maquet, outside the submitted work. G. Bellani has nothing to disclose. T. Pham has nothing to disclose. L. Brochard reports grants from Medtronic Covidien (for research on proportional assist ventilation), non-financial support from Air Liquide (for research on cardiopulmonary resuscitation), non-financial support from Philips and Sentec (for research on sleep), grants and non-financial support from Fisher Paykel (for research on high flow), and personal fees from Baxter (for consulting), outside the submitted work. J.G. Laffey has nothing to disclose.

Support statement: Funding was received from the European Respiratory Society and the European Society of Intensive Care Medicine. Funding information for this article has been deposited with the Crossref Funder Registry.

\section{References}

1 Boles JM, Bion J, Connors A, et al. Weaning from mechanical ventilation. Eur Respir J 2007; 29: 1033-1056.

2 Esteban A, Ferguson ND, Meade MO, et al. Evolution of mechanical ventilation in response to clinical research. Am J Respir Crit Care Med 2008; 177: 170-177.

3 McConville JF, Kress JP. Weaning patients from the ventilator. N Engl J Med 2012; 367: 2233-2239.

4 Fagon JY, Chastre J, Vuagnat A, et al. Nosocomial pneumonia and mortality among patients in intensive care units. JAMA 1996; 275: 866-869.

5 Provost KA, El-Solh AA. Complications associated with mechanical ventilation. In: Tobin MJ, ed. Principles and Practice of Mechanical Ventilation. 3rd Edn. New York, McGraw-Hill, 2013; pp. 973-994.

6 Epstein SK, Ciubotaru RL. Independent effects of etiology of failure and time to reintubation on outcome for patients failing extubation. Am J Respir Crit Care Med 1998; 158: 489-493.

7 Thille AW, Richard JC, Brochard L. The decision to extubate in the intensive care unit. Am J Respir Crit Care Med 2013; 187: 1294-1302.

8 Blackwood B, Alderdice F, Burns K, et al. Use of weaning protocols for reducing duration of mechanical ventilation in critically ill adult patients: Cochrane systematic review and meta-analysis. BMJ 2011; 342: c7237.

9 Ely EW, Baker AM, Dunagan DP, et al. Effect on the duration of mechanical ventilation of identifying patients capable of breathing spontaneously. N Engl J Med 1996; 335: 1864-1869.

10 Girard TD, Kress JP, Fuchs BD, et al. Efficacy and safety of a paired sedation and ventilator weaning protocol for mechanically ventilated patients in intensive care (Awakening and Breathing Controlled trial): a randomised controlled trial. Lancet 2008; 371: 126-134.

11 Béduneau G, Pham T, Schortgen F, et al. Epidemiology of weaning outcome according to a new definition. The WIND Study. Am J Respir Crit Care Med 2017; 195: 772-783.

12 Brightling C, Genton C, Bill W, et al. ERS Clinical Research Collaborations: underpinning research excellence. Eur Respir J 2018; 52: 1801534.

13 Bellani G, Laffey JG, Pham T, et al. Epidemiology, patterns of care, and mortality for patients with acute respiratory distress syndrome in intensive care units in 50 countries. JAMA 2016; 315: 788-800.

14 Burns KEA, Misak C, Herridge M, et al. Patient and family engagement in the ICU. Untapped opportunities and underrecognized challenges. Am J Respir Crit Care Med 2018; 198: 310-319.

15 Laffey JG, Madotto F, Bellani G, et al. Geo-economic variations in epidemiology, patterns of care, and outcomes in patients with acute respiratory distress syndrome: insights from the LUNG SAFE prospective cohort study. Lancet Respir Med 2017; 5: 627-638. 